\title{
Concepción de "extensión rural" de los extensionistas rurales argentinos que trabajan en el sistema público nacional con pequeños productores
}

Fernando Landini*

Recibido: 2014-04-08 Aprobado: 2014-10-08 Disponible en línea: 2015-15-03

doi:I0.III44/Javeriana.cdrı2-75.cere

Cómo citar este artículo: Landini, F. (2015). Concepción de "extensión rural" de los extensionistas rurales argentinos que trabajan en el sistema público nacional con pequeños productores. Cuadernos de Desarrollo Rural, ${ }_{22}(75)$, 33-53. http://dx.doi.org/10.1II44/Javeriana.cdri2-75.cere

\footnotetext{
* Doctor en Psicología (Universidad de Buenos Aires). Magister en Desarrollo Rural (Universidad Politécnica de Madrid). Magister en Desarrollo Local (Universidad Internacional de Andalucía). Investigador Asistente (Consejo Nacional de Investigaciones Científicas y Técnicas). Investigador Superior (Universidad de la Cuenca del Plata). Correo electrónico: landini_fer@hotmail.com
} 


\title{
Resumen
}

En vista de que las concepciones de extensión guían las acciones de los extensionistas en terreno, se realizó una encuesta vía correo electrónico a gestores argentinos que trabajan en el ámbito público nacional. Se obtuvieron 219 respuestas a preguntas abiertas que fueron categorizadas y cuantificadas. La concepción de extensión observada no corresponde con los modelos teóricos y se observa la articulación de elementos correspondientes a diferentes modelos. Se destaca la importancia del trabajo grupal y la participación en el contexto del trabajo con productores, la existencia de una concepción amplia de desarrollo y la propuesta de trabajar de manera interdisciplinaria en equipos de extensión.

\section{Palabras clave:}

extensión difusionista; modelos de extensión; prácticas de extensión rural; psicología del extensionista

\section{The Conception of "Rural Extension" of the Argentinian Rural Extensionists Working in the National Public System with Small Producers}

\begin{abstract}
We performed a survey via email to Argentinian extensionists that work in the national public sphere, given that the conceptions of extension guide the actions of extensionists in the field. We obtained 219 answers; open questions were categorized and quantified. The observed conception of extension does not correspond to the theoretical models and it can be seen there is articulation between elements corresponding to different models. The highlights include the importance of teamwork and participation, in the context of work with producers; the existence of a broad conception of development; and the proposal of interdisciplinary work on extension teams.
\end{abstract}

Keywords:

diffusionist extension; extension models; rural extension practices; extentionist psychology 


\section{Conception de la vulgarisation rurale des agents de vulgarisation argentins qui travaillent dans le système public avec les petits producteurs}

\section{Résumé}

Etant-donné que les conceptions de vulgarisation dirigent les actions des agents de vulgarisation dans le terrain, on a réalisé une enquête par le courrier électronique aux agents de vulgarisation argentins qui travaillent dans le domaine public national. On a obtenu 2I9 réponses. Les questions ouvertes ont étés catégorisées et quantifiées. La conception de vulgarisation observée n’est pas ajustée aux modèles théoriques, et on observe l'articulation d'éléments correspondants aux différents modèles. On souligne l'importance du travail en groupe et la participation dans le contexte du travail avec les producteurs, l'existence d'une conception élargie du développement et la proposition de travailler d'une manière interdisciplinaire avec les équipes de vulgarisation.

Mots-clés:

vulgarisation diffusionniste; modèles de vulgarisation; pratiques de vulgarisation rurale; psychologie de l'agent de vulgarisation

\section{Concepção de "extensão rural" dos extensionistas rurais argentinos que trabalham no sistema público nacional com pequenos produtores}

\section{Resumo}

Em vista de que as concepções de extensão orientam as ações em campo dos extensionistas, um inquérito via correio-eletrônico a gestores argentinos que trabalham no âmbito público nacional foi realizado. Obtiveram-se 219 respostas a perguntas abertas que foram categorizadas e quantificadas. A concepção de extensão observada não corresponde com os modelos teóricos e evidencia-se a articulação de elementos correspondentes a diferentes modelos. Releva-se a importância do trabalho grupal e a participação no contexto do trabalho com produtores, a existência de uma concepção ampla de desenvolvimento e a proposta de trabalhar de maneira interdisciplinar em equipes de extensão.

Palavras-chave:

extensão difusionista; modelos de extensão; práticas de extensão rural; psicologia do extensionista 


\section{Introducción}

La extensión rural (ER) constituye una herramienta de gran potencial para favorecer procesos de desarrollo rural (McLeod Rivera y Qamar, 2003). Esto cobra particular relevancia en América Latina, donde se asiste a un resurgimiento o retorno de la ER pública, después de los procesos privatizadores de fines del siglo pasado (Alemany y Sevilla Guzmán, 2007). En este contexto, resulta llamativo que la ER no aparezca hoy como un objeto prioritario de indagación empírica o de investigación sistemática.

Si se revisa la literatura, se observa la existencia de múltiples trabajos que discuten cómo debería trabajarse en extensión o cuáles son los modelos de ER que deberían utilizarse (por ejemplo, Landini, Murtagh y Lacanna, 2009; Machado, de Hegedüs y Silveira, 2006), trabajos orientados a caracterizar o definir la agricultura familiar, el campesinado o alguna categoría asociada (por ejemplo, Tort y Román, 2005; Tsakoumagkos, Soverna y Craviotti, 2000). También, investigaciones sobre estrategias de subsistencia y lógicas campesinas (por ejemplo, Cáceres, 1995; Patiño, 2000; Silvetti y Cáceres, 1998; Stage y Rekve, 1998), estudios referidos a saberes locales (por ejemplo, Landini, 20ıо; Mora Delgado, 2008; Núñez, 2004) o sobre procesos asociativos en el contexto de la pequeña producción agropecuaria (por ejemplo, Elgue y Chiaradía, 2007; Lobos, 2005).

Así, si bien la agricultura familiar, en tanto público privilegiado de la ER, aparece como claro objeto de investigación, junto con trabajos de discusión y reflexión sobre enfoques y metodologías de extensión, lo que tiene una presencia sustancialmente menor, son trabajos empíricos que aborden la ER como objeto de investigación. En consecuencia, se hace evidente la necesidad de fortalecer la investigación sobre ER y sobre el impacto diferencial de diferentes modos de ponerla en práctica desde una perspectiva empírica y crítica, que no parta de lo que debería ser sino del estudio de lo que es, con el fin de trabajar por aquello que queremos que sea.

En este contexto cobra interés una propuesta reciente surgida en América Latina, que se propone estudiar los fundamentos psicosociales de los procesos de desarrollo rural, con énfasis en la ER (Landini, Bianqui y Russo, 2013; Landini, Leeuwis, Long y Murtagh, 20I4; Landini, Long, Leeuwis y Murtagh, 20I4). Esta propuesta, que busca articularse con el Abordaje Orientado al Actor (Long, 2007), sostiene la necesidad de analizar la articulación psicosocial de la agencia humana para dar cuenta de los procesos colectivos, a la vez que se evita utilizar supuestos del sentido común sobre el funcionamiento del psiquismo para explicar procesos sociales. 
En este trabajo se parte de la premisa de que las concepciones sobre la realidad y sobre los objetos sociales guían las prácticas de los diferentes actores sociales (Elcherothpops, Doise y Reicher, 20II; Howarth, 2006), en tanto estas delinean objetivos, valores y prioridades para la acción que se convierten en conductas con atención a la constricciones sociales y materiales a las que se enfrentan (Landini, 20II; Landini, Long, Leeuwis y Murtagh, 20I4). Así, si se desea estudiar y comprender las prácticas de ER, no desde una perspectiva del hacer ideal sino desde lo que sucede empíricamente, queda en un primer plano la indagación de las concepciones que los propios extensionistas tienen de esta y de sus objetivos.

A los fines analíticos, en este trabajo se toman cuatro modelos de ER que, si bien en el plano teórico pueden diferenciarse, en la práctica suelen aparecer articulados o incluso superpuestos. Primero, la ER difusionista, importada de Estados Unidos a mediados del siglo pasado, centrada en la transferencia de tecnologías de expertos a productores (Tort, 2008). Este enfoque posee como principales características la desvalorización del saber empírico del productor y el establecimiento de relaciones jerárquicas entre extensionistas y beneficiarios (Landini et al., 2009). Desde fines de los años sesenta del siglo XX este modelo fue cuestionado y fue propuesto el establecimiento de relaciones horizontales entre extensionistas y productores, orientadas a la construcción conjunta de acciones y saberes superadores (Freire, 1973). Esto ha sido denominado ER crítica (Tommasino, González, Guedes y Prieto, 2006).

Con el nuevo siglo, dos nuevas formas de pensar la ER cobran fuerza. Por un lado, el enfoque territorial de la ER, que implica pensar la ER sin focalizar en rubros productivos sino desde los procesos de articulación social e institucional que se dan en espacios locales (Instituto Nacional de Tecnología Agropecuaria [INTA], 2004). Por último, también encontramos el abordaje de los sistemas de innovación. En este caso, el rol del extensionista queda ubicado en la facilitación de procesos de innovación a partir de la articulación entre diferentes actores: productores, comerciantes, industria, centros de investigación, sector público, etcétera (Leeuwis, 2004), pero con independencia del enfoque territorial.

En este contexto, con base en los diferentes enfoques mencionados, en este trabajo se presentan los resultados de una investigación orientada a describir la concepción de ER de una muestra de extensionistas argentinos que trabajan en el sistema público nacional con pequeños productores. 


\section{Metodología}

Se realizó una investigación exploratoria-descriptiva de carácter cualitativocuantitativo basada en la realización de una encuesta por correo electrónico a extensionistas argentinos que trabajan en instituciones públicas de extensión de escala nacional. La muestra fue no probabilística incidental. Visto lo anterior, se destaca que los resultados no son necesariamente representativos de la ER en Argentina, tanto porque se incluyeron solo instituciones públicas de escala nacional, como porque la muestra no fue construida mediante procedimientos probabilísticos. Se obtuvieron 2I9 respuestas a la encuesta: I43 hombres y 76 mujeres; 106 del INTA; 72 del Programa ProHuerta (implementado conjuntamente por el Ministerio de Desarrollo Social y el INTA); y 4i de la Subsecretaría de Agricultura Familiar (SAF) del Ministerio de Agricultura.

Dado que quienes trabajan en ProHuerta también pueden hacerlo en otras acciones de INTA, se categorizó como "ProHuerta” a aquellos que dedican a este programa 50\% o más de su tiempo y como "INTA" a quienes no lo hacen. Al sumar la cantidad de extensionistas que trabajan en estas instituciones, se calcula que la muestra corresponde a 9\% del total. Para la obtención de las respuestas se contactó a autoridades de diferentes escalas territoriales de las instituciones implicadas, con el objetivo de obtener colaboración con el reenvío por correo electrónico de los formularios.

La encuesta realizada forma parte de una investigación más amplia orientada a alcanzar objetivos que exceden este trabajo. La primera parte incluye preguntas cerradas referidas a variables como: sexo, edad, máximo nivel educativo, título académico, institución en la que trabaja actualmente, región del país donde desempeña actividades y años de experiencia como extensionista.

La segunda parte incluyó preguntas abiertas sobre los principales problemas que tienen los pequeños productores para progresar y salir adelante y las dificultades que surgen en el trabajo de ER. A continuación, se indagó, como pregunta cerrada, si la psicología podría contribuir o no a resolver algunos de esos problemas. En caso de que la respuesta fuese afirmativa se preguntaba cuáles serían las dificultades con las cuales podría contribuir, cuáles otros aportes podría hacer y cómo se podrían concretar. En caso de que la respuesta fuese negativa, se indagaron las razones por las cuales se consideraba así.

Podría argumentarse que estas preguntas no se incluyen ninguna específica que indague lo que se considera ER. No obstante, una pregunta directa hubiera 
obtenido preferentemente respuestas apoyadas en aquello que los encuestados suponen que los encuestadores esperan escuchar o, en el mejor de los casos, creencias conscientes de tipo discursivo de lo que se piensa que es ER. Así, la investigación se hubiera malogrado al no atender a los supuestos, generalmente implícitos, que guían las prácticas.

Por el contrario, se considera que las preguntas realizadas llevan indirectamente a los encuestados a hablar de ER, de modo que expresaran sus supuestos, situación que permite captarlos en un contexto más espontáneo. No obstante, se reconoce que otras preguntas podrían haber hecho aparecer otras respuestas o llevado a encontrar diferentes énfasis, lo que lleva a tener en cuenta que los resultados obtenidos dependen en parte del contexto en que fueron producidos.

Para analizar las encuestas se utilizaron dos tipos de software Atlas Ti y SPSS. El primero fue utilizado para categorizar fragmentos de las respuestas, a partir de ejes temáticos surgidos del discurso de los encuestados o planteados como dimensiones de análisis relevantes de acuerdo con los diferentes modelos de ER mencionados en la introducción; por tanto, en este proceso se construyeron definiciones provisorias de cada una de las categorías utilizadas. A continuación se realizaron nuevas lecturas del material, con el fin de categorizar fragmentos incluidos en las definiciones de cada categoría que no hubieran sido notados en un principio.

En este proceso se llegó a definiciones consolidadas de cada categoría. En sucesivos momentos de categorización se optó por incluir dentro de las categorías aquellos fragmentos dudosos, para que ninguno quedara por fuera. Finalmente, se terminó de ajustar la definición de la categoría, con criterios para definir sobre casos dudosos; esta definición fue utilizada para hacer una revisión de todos los fragmentos incluidos en cada categoría y excluir aquellos que no correspondieran a las definiciones finales.

En este contexto se optó por dividir estas categorías en tres tipos. Aquellas en las cuales la definición final de la categoría permitió establecer criterios claros y confiables de inclusión/exclusión; categorías con definiciones consistentes pero que presentaban en el momento final dudas en cuanto a la inclusión de diversos fragmentos; y categorías con límites difusos utilizadas simplemente para identificar temas de interés. Se destaca que en este proceso no se analizaron de manera diferenciada las respuestas a cada pregunta, sino que se procuró identificar en general las concepciones de extensión subyacentes a los textos producidos por los encuestados. Por esta razón se informan resultados globales y no por pregunta. 
Los tres tipos de categorías mencionados fueron incluidos en el SPSS, y cada categoría surgida inductivamente fue considerada como variable. Las categorías del primer tipo fueron analizadas como variables ordinales, según la cantidad de fragmentos que aparecían en cada encuesta. Las segundas fueron tomadas como nominales dicotómicas (presencia/ausencia); la variable se consideró como "presente" cuando había sido mencionada al menos una vez por el entrevistado. Se decidió utilizar ambos tipos de variables para hacer cuantificaciones simples (análisis de frecuencia), mientras que solo se trabajó con asociaciones estadísticas con las del primer grupo.

Para finalizar, las del tercer tipo solo se utilizaron para el análisis cualitativo. Cuando se analizó la asociación entre las categorías del primer tipo y variables nominales se optó recategorizarlas como nominales (presencia/ausencia), y para ello se usó Chi Cuadrado $\left(\chi^{2}\right)$. Cuando la relación se estableció con variables de nivel ordinal, intervalar o de razón, se utilizó el coeficiente de correlación de Spearman.

\section{Resultados}

\section{I. Descripción cuantitativa de la concepción de la extensión}

En la Tabla I se indica la cantidad de encuestas, sobre las 219 obtenidas, en que se mencionan los contenidos listados a la izquierda. Solo se indican aquellas categorías mencionadas por más de 1o\% de los encuestados, correspondientes al primer y segundo tipo de variables, según se los definió previamente. En el caso de las variables de nivel ordinal, en esta tabla se las cuantifica como dicotómicas (presencia-ausencia) con fines expositivos.

$\mathrm{Al}$ existir una cantidad importante de contenidos entre $10 \%$ y $20 \%$, podría pensarse que se trata de cuestiones que poseen escasa relevancia en la perspectiva de los extensionistas. No obstante, se debe tener presente que se trata de menciones espontáneas, razón por la cual el uso de otros formatos de investigación, como escalas tipo Likert, sin duda arrojaría porcentajes más altos. Por esto, parece recomendable tomar los distintos ítems listados como indicadores del hecho de su presencia y de su importancia relativa respecto a otros contenidos, más que como valores absolutos de su nivel de presencia en la población encuestada. 
TABla l. Elementos que componen la concepción de extensión de los encuestados, según áreas temáticas

Contenidos

Frecuencia Porcentaje

A. Acciones implicadas en la tarea de EXtensión Rural

\begin{tabular}{|c|c|c|}
\hline 1-Gestión grupal, manejo de conflictos y fortalecimiento de vínculos & 133 & $60.7 \%$ \\
\hline 2- Hacer diagnósticos, planificación, seguimiento y evaluación de proyectos & II6* & $53 \%$ \\
\hline 3-El trabajo de ER debe ser interdisciplinario & 79 & $36.1 \%$ \\
\hline 4- La labor de ER requiere formar equipos de trabajo & 66 & $30.1 \%$ \\
\hline 5- La ER implica capacitar, enseñar o formar al productor & 37 & $16.9 \%$ \\
\hline 6- Gestionar o desarrollar trabajos o acciones participativas & 36 & $\mathrm{I} 6.4 \%$ \\
\hline \multicolumn{3}{|l|}{ B. ENFOQUE O LINEAMIENTOS FUNDAMENTALES } \\
\hline $\begin{array}{l}\text { 7-Se valora la participación y el tener en cuenta las } \\
\text { necesidades, intereses, cultura y racionalidad campesina }\end{array}$ & 99 & $45.2 \%$ \\
\hline $\begin{array}{l}\text { 8- Enfoque integral de la ER y el desarrollo, no solo } \\
\text { productivo, sino también con dimensión social }\end{array}$ & 67 & $30.6 \%$ \\
\hline 9- La ER implica articulaciones interinstitucionales & 52 & $23.7 \%$ \\
\hline $\begin{array}{l}\text { Io- ER como cambio de mentalidad, o como } \\
\text { transferencia/adopción de tecnologías }\end{array}$ & $4 \mathrm{I}$ & $18.7 \%$ \\
\hline $\begin{array}{l}\text { II -Vínculo horizontal técnico-productor, construcción } \\
\text { consensuada, igualdad de saberes, rechazo al transferencismo }\end{array}$ & 36 & $16.4 \%$ \\
\hline \multicolumn{3}{|l|}{ C. VÍNCULO TÉCNICO-PRODUCTOR Y SABERES DE LOS PRODUCTORES } \\
\hline $\begin{array}{l}\text { I2- Los pequeños productores poseen una } \\
\text { racionalidad diferente a la del extensionista }\end{array}$ & 47 & $2 \mathrm{I} .5 \%$ \\
\hline $\begin{array}{l}\text { I3- Importancia de la comunicación y capacidad de } \\
\text { llegada al productor, necesidad de buen vínculo }\end{array}$ & 45 & $20.5 \%$ \\
\hline I4- Necesidad de comprender al productor & $4 \mathrm{I}$ & $\mathrm{I} 8.7 \%$ \\
\hline I5-Desvalorización del saber o las prácticas productivas campesinas & 39 & $17.8 \%$ \\
\hline $\begin{array}{l}\text { I6- Reconocimiento y valoración del saber campesino, } \\
\text { de su trabajo y de sus capacidades }\end{array}$ & 30 & $13.7 \%$ \\
\hline \multicolumn{3}{|l|}{ D. Otros } \\
\hline $\begin{array}{l}\text { I7- Se espera/requiere una actitud proactiva/participativa } \\
\text { de los productores, comprometida con el desarrollo }\end{array}$ & $80^{*}$ & $36.5 \%$ \\
\hline I8-Se necesita de técnicas y herramientas para trabajar en ER & 64 & $29.2 \%$ \\
\hline 19- Los extensionistas necesitan recibir capacitación & $62^{*}$ & $28.3 \%$ \\
\hline $\begin{array}{l}\text { 20- Percepción crítica en relación con la noción de } \\
\text { desarrollo, ER y saber científico, entre otras }\end{array}$ & 54 & $24.7 \%$ \\
\hline 2I- ER no es asistencialismo ni clientelismo & $32^{*}$ & $\mathrm{I} 4.6 \%$ \\
\hline 22- Expectativa de visión y práctica empresarial & 27 & $12.3 \%$ \\
\hline 23- Generar autonomía y autogestión y desarrollar recursos y capacidades & $24^{*}$ & $11 \%$ \\
\hline
\end{tabular}

* Los contenidos con asterisco corresponden a variables con nivel de medición nominal (del segundo tipo antes mencionado), mientras que el resto posee nivel ordinal.

FuENTE: elaboración propia 


\subsection{Descripción cualitativa de la concepción de la extensión}

A continuación se describe la concepción de ER de los encuestados a nivel cualitativo, según los diferentes ejes temáticos en que se han agrupado las variables. El primero (A), refiere a las acciones implicadas en hacer ER. Se destaca aquí el trabajo con grupos de productores, el fortalecimiento de los vínculos y la gestión de conflictos (60.7\%). En esta línea se menciona "la organización de actividades en forma asociada" y "la aplicación de metodologías de grupo" como elemento fundamental de la ER. Esto cobra sentido si se tiene en cuenta que en la Argentina la mayor parte de las acciones de ER se orientan al trabajo con grupos de productores.

Llama la atención que si bien la mayoría de los encuestados era graduado en ingeniería agrónoma, un elemento importante de su accionar apareció como externo a su formación. Luego, 53 \% de los encuestados relacionan la ER con la gestión de proyectos, lo que incluye diagnosticar, planificar, dar seguimiento y evaluar, aunque lo más usual es la mención de la idea de "proyecto", lo que evidencia que, en lo institucional, la mayor parte de las acciones de ER se articulan como "proyectos".

Otras dos categorías también se incluyen en el área del accionar de la ER. Un I6.9\% de los encuestados menciona dar capacitación a los productores, por ejemplo, “charlas técnicas" o "un proceso de promoción, capacitación”. Posiblemente, el bajo porcentaje se deba a que se trata de una labor percibida como poco problemática, por tanto, apareció pocas veces en las respuestas.

Por último, la tarea de ER también involucra la gestión de acciones participativas ( $16.4 \%)$. Como dijo una encuestada: hay que "aprender a valorar las percepciones de los productores, aprender que las instancias sean participativas”. Es notorio el bajo porcentaje de referencias, puesto que la participación aparece en múltiples discursos de ER como aspecto fundamental, y en este caso no se puede argumentar que para los profesionales técnicos se trate de un enfoque poco problemático.

En cuanto a las acciones de ER, los encuestados también destacan la necesidad de trabajar de manera interdisciplinaria (36.I\%) formando equipos de ER (30.1\%), lo que es mencionado, por lo general, como una falta o un problema: "Otra dificultad importante es la falta de equipos interdisciplinarios”. Así, se entrevé que los extensionistas perciben la complejidad de su práctica, que no solo incluye aspectos técnico-productivos sino también cuestiones comerciales, organizativas y, en general, sociales, razón por la que desean equipos interdisciplinarios de ER. 
Por otra parte, resulta de gran interés identificar el enfoque o los lineamientos fundamentales a partir de los cuales los encuestados piensan la ER (B). En este sentido, un importante porcentaje de los encuestados (45.2\%) valoran en las acciones de ER la participación o el tener en cuenta las necesidades, intereses o punto de vista de los beneficiarios. Queda claro que ambas cuestiones no son lo mismo, ya que pueden tenerse en cuenta las necesidades y punto de vista de los productores pero sin generar procesos participativos. No obstante, ambas refieren a un núcleo común: tomar en cuenta las necesidades o prioridades del territorio o de los productores con que se trabaja. En general, este aspecto de la concepción de la ER se expresa a partir de la descripción de problemas o de aquello que faltaría en el trabajo de ER. Como señaló otra persona encuestada: "Muchas veces no se logra tener resultado porque estos proyectos, en su mayoría, son pensados por las instituciones sin participación de los supuestos beneficiarios. Es importante la construcción ‘desde abajo’ de los proyectos para tener objetivos en línea con las necesidades de las personas”.

Resulta interesante señalar el contraste entre el $16.4 \%$ que afirma como parte de la ER la gestión de acciones participativas y el $45.2 \%$ que valora la participación y el tomar en cuenta las necesidades y cultura de los beneficiarios. Esto sugiere que la idea de participación o reconocimiento del punto de vista del productor aparece más como una queja, referida a su falta en los encuadres institucionales o en los proyectos de ER, y no tanto como una labor del extensionista, quien en mucha menor medida se percibe responsable de favorecer esa participación.

También se observa que $30.6 \%$ de los encuestados propone o posee una concepción de la ER o del desarrollo que no es solo técnica o productiva, sino que incorpora la dimensión social y humana. Esto supone reconocer la complejidad de la ER. Dentro de este rubro, la mayor parte de los fragmentos implican una crítica a una visión exclusivamente productiva del desarrollo: "Los agrónomos tenemos una visión productivista [...] y dejamos de lado los factores sociológicos que le pasan a la persona”. De esto se deriva la necesidad de un abordaje multidisciplinar, que pueda atender la complejidad de la realidad para generar procesos de desarrollo integrantes y no "crecimiento productivo pero sin desarrollo".

De acuerdo con los lineamientos utilizados por los encuestados para pensar la ER, también aparecen referencias a la necesidad de generar procesos de articulación interinstitucional (23.7\%), una propuesta en línea con los esquemas que hablan de desarrollo local o territorial. En cualquier caso, sí son pocos los que mencionan de forma explícita la idea de un abordaje territorial (7.3\%), algo mucho más conceptual que la propuesta operativa de articulación interinstitucional. 
Por su parte, la discusión entre una ER orientada a la transferencia de tecnologías y al cambio de mentalidad de los productores, y una ER horizontal y dialógica, aparece con bajos porcentajes, algo que contrasta con la importancia que diferentes estudiosos dan al tema. En efecto, solo I $8.7 \%$ de los encuestados habla de transferencia de tecnologías, mientras que i6.4\% se pueden categorizar como dialógicos, según la terminología freireana. ¿Será que la discusión entre ambos enfoques debiera considerarse saldada o superada? De cualquier manera, se trata de una cuestión que aparece con frecuencia en discusiones o espacios de capacitación de extensionistas en Argentina.

En cuanto al último punto, también en relación con los lineamientos que organizan la concepción de la ER, existen dos áreas que destacan por su escasa presencia. Por un lado, solo $7.3 \%$ señala que la ER implica prestar atención al área de género/mujer y juventud. Por otro parte, solo $3.6 \%$ se planteó la figura del extensionista como facilitador o dinamizador de procesos, en lugar de como gestor, una figura acorde con los modelos actuales de desarrollo territorial o de sistemas de innovación (Leeuwis, 2004).

Por su parte, también existe un grupo de categorías que aborda la cuestión del vínculo técnico-productor y los saberes de los productores (C). En primer lugar, se observa un conjunto de fragmentos según los cuales los pequeños productores poseen una racionalidad o una forma de pensar diferente a la de los extensionistas (21.5\%). Como explica un entrevistado: "No revaloramos las experiencias, conocimientos y lógicas de solución de los problemas que tienen los pequeños productores”, de lo que se sigue la necesidad de respetar "la visión de las potenciales poblaciones objetivo, quitarles el sesgo de racionalidades de los técnicos y de las instituciones, que priorizan visiones y objetivos propios”. Esto se relaciona con múltiples comentarios que señalan la dificultad (e importancia) de comprender a los pequeños productores (I8.7\%), a partir de lo cual se plantea un pedido a la psicología de "ayudarnos a los extensionistas a tratar de entender mejor la psicología del productor”. Así, en estas dos categorías temáticas, se observa el reconocimiento de que los pequeños productores poseen una forma de pensar o comprender que no se identifica con la del extensionista.

En esta línea, $13.7 \%$ de los encuestados reconoce y valora el saber campesino, su modo de trabajo o sus capacidades. Se comenta que hay que "valorizar los saberes, las experiencias y los recursos del pequeño productor”. En contraste, $17.8 \%$ critica el saber o el modo de hacer de los beneficiarios a nivel productivo, al afirmar, por ejemplo "escasa diversificación de la producción” o "bajo nivel cultural referido al grado de capacitación en la actividad que realizan”. Entonces, se observa por un lado 
un reconocimiento productivo y de las capacidades de los productores, mientras que por el otro encontramos críticas al modo de producir y organizar la producción, situación que se expresa en diferentes formas de hacer ER.

También se señala la importancia de tener una buena comunicación técnicoproductor, es decir, tener capacidad de llegada con la gente (20.5\%), por eso se plantea que "el extensionista debe tener un carisma y llegada especial para este sector de productores". Ahora bien, lo que llama la atención es que, pese a destacarse la importancia de "llegar" a los productores, poco se habla de la necesidad de desarrollar capacidades educativas o pedagógicas para llevar adelante acciones de ER, tema solo mencionado por $3.2 \%$ de los casos.

Para finalizar, también existen varios contenidos, clasificados dentro de Otros (D), que vale mencionar. En relación con los productores, $36.5 \%$ de los encuestados sostiene que es necesario adoptar una actitud proactiva o participativa, comprometida con el trabajo de ER. Así, se reconoce que el extensionista necesita de los beneficiarios para obtener un buen resultado en las tareas de ER, pues este compromiso se considera como la contrapartida de la ayuda que se les estaría brindando. Esta expectativa de los extensionistas incluye la asistencia a reuniones, la adopción de tecnologías propuestas y el uso de insumos entregados, entre otras cuestiones. Se pide entonces a los productores "compromiso con la propuesta de extensión”, muchas veces como consecuencia de percibir escaso compromiso.

Los encuestados también señalan que la ER requiere de técnicas y herramientas específicas (29.2\%), lo que se relaciona con la idea de que es necesario recibir capacitación (28.3\%). Una articulación de estos dos ejes puede verse cuando se señala que especialistas en el área social podrían "brindar métodos [dar formación] para la construcción grupal de diagnósticos, soluciones y formas de trabajo”, o “aportar metodologías para facilitar los trabajos de diagnóstico".

Es significativo el porcentaje de extensionistas, $24.7 \%$, que posee un posicionamiento crítico sobre lo que hace, al poner en cuestión concepciones tradicionales de "desarrollo" y "extensión” o el mismo estatus del conocimiento científico. Un ejemplo puede observarse en la siguiente cita: "Los pequeños productores no logran lo resultados que ‘esperan’ porque la génesis de la idea de desarrollo ya es complicada y mas aun las formas que 'hemos' encontrado para canalizar una idea que desde el vamos no nos convence del todo".

Esta actitud crítica y reflexiva resulta interesante, ya que surgió de manera espontánea en casi un cuarto de las encuestas, lo que sugiere capacidad para repensar las propias prácticas, es decir, repensar el modo de hacer ER. 


\subsection{Relaciones estadísticas entre contenidos y diversas variables}

Se estudiaron las relaciones estadísticas entre los contenidos de la concepción de ER cuantificados como variables ordinales (ver Metodología) y las variables: sexo, edad, institución de pertenencia, región donde se desempeñan, nivel educativo, título académico y experiencia en el trabajo de ER. El objetivo de este análisis fue identificar la existencia de perfiles diferenciales.

Tabla 2. Relación entre elementos de la concepción de extensión y diferentes variables

\begin{tabular}{|c|c|c|c|c|c|c|c|}
\hline \multirow{3}{*}{ Contenidos } & \multirow{3}{*}{$\begin{array}{l}\text { Sexo } \\
\chi^{2}=\end{array}$} & \multirow{3}{*}{$\begin{array}{l}\text { EDAD } \\
r s=\end{array}$} & \multirow{3}{*}{$\begin{array}{l}\text { EXPERIENCIA } \\
r s=\end{array}$} & \multirow{3}{*}{$\begin{array}{l}\text { INSTITUCIÓN } \\
\chi^{2}=\end{array}$} & \multirow{3}{*}{$\begin{array}{l}\text { REGIÓN } \\
\chi^{2}\end{array}$} & \multirow{3}{*}{$\begin{array}{l}\text { Nivel } \\
\text { EDUCATIVO } \\
r s=\end{array}$} & \multirow{3}{*}{$\begin{array}{l}\text { Título } \\
\chi^{2} \\
\text { / Coef. }=\end{array}$} \\
\hline & & & & & & & \\
\hline & & & & & & & \\
\hline I & 2,89(I) & $-0,2 \mathrm{I}^{* *}$ & $-0,10$ & $0,31(2)$ & $0,76(4)$ & $0,16^{*}$ & $\mathrm{II}, 4 \mathrm{O}(4)^{*}$ \\
\hline 3 & $\mathrm{I}, \mathrm{I} 2(\mathrm{I})$ & 0,04 & 0,10 & $7,03(2)^{*}$ & $5, \circ 4(4)$ & $0,24^{* *}$ & $9, \mathrm{I} 6(4)$ \\
\hline 4 & $0,42(\mathrm{I})$ & $-0,04$ & 0,03 & $7,79(2)^{*}$ & $4,99(4)$ & $0,17^{* *}$ & $13,38(4)^{*}$ \\
\hline 5 & 0,49 (I) & 0,04 & 0,04 & II, $56(2)^{* *}$ & $6,70(4)$ & $-0, \mathrm{I} 2$ & 0,10 \\
\hline 6 & $\mathrm{I}, 80(\mathrm{I})$ & $-0,03$ & 0,06 & $9,53(2)^{* *}$ & $5,93(4)$ & $0,16^{*}$ & $0, \mathrm{I} 2$ \\
\hline 7 & $0,57(\mathrm{I})$ & 0,02 & $0,14^{*}$ & $\mathrm{I}, 23(2)$ & $\mathrm{I}, 37(4)$ & $0,18^{*}$ & $9,48(4)$ \\
\hline 8 & $0,29(\mathrm{I})$ & $0,0 \mathrm{I}$ & 0,08 & $3,55(2)$ & $4,82(4)$ & $0,20^{* *}$ & $8,42(4)$ \\
\hline 9 & $5,38(\mathrm{I})^{*}$ & $-0,03$ & $0,0 \mathrm{I}$ & $2,36(2)$ & $2,02(4)$ & $0,19^{* *}$ & $6,09(4)$ \\
\hline IO & $\mathrm{I}, 38(\mathrm{I})$ & $0, \mathrm{II}$ & $0,19^{* *}$ & $\mathrm{I}, 46(2)$ & $2,97(4)$ & 0,03 & 0,18 \\
\hline II & $\mathrm{I}, 80(\mathrm{I})$ & 0,04 & 0,04 & $3,40(2)$ & $5,90(4)$ & $0,2 \mathrm{I}^{* *}$ & 0,20 \\
\hline I2 & 2,63(I) & 0,06 & 0,05 & $0,9 \mathrm{I}(2)$ & $0,56(4)$ & 0,13 & $0,22^{*}$ \\
\hline 13 & $2,37(\mathrm{I})$ & 0,07 & $-0,04$ & $3,03(2)$ & $2,35(4)$ & $0,15^{*}$ & $0, \mathrm{I} 4$ \\
\hline I4 & $3,02(\mathrm{I})$ & $-0,03$ & $-0,02$ & $2,29(2)$ & $3,60(4)$ & O,OI & 0,09 \\
\hline 15 & $0,03(\mathrm{I})$ & $0, \infty 0$ & 0,00 & $2,98(2)$ & $2, \mathrm{I} 2(4)$ & 0,02 & 0,12 \\
\hline I6 & $0,34(\mathrm{I})$ & 0,07 & $0,16^{*}$ & $\mathrm{I}, \mathrm{I} 2(2)$ & $2,24(4)$ & $0,22^{* *}$ & 0,18 \\
\hline I8 & $0,3 \mathrm{I}(\mathrm{I})$ & $-0,07$ & $-0,07$ & $4,8 \mathrm{I}(2)$ & $4,78(4)$ & 0,06 & $\mathrm{II}, \mathrm{IO}(4)^{*}$ \\
\hline 20 & $0,33(\mathrm{I})$ & 0,07 & 0,08 & $8,97(2)^{*}$ & $7,9 \mathrm{I}(4)$ & $0,14^{*}$ & 0,18 \\
\hline 22 & $0,07(\mathrm{I})$ & 0,08 & 0,04 & $2,76(2)$ & $0,82(4)$ & $-0,0 \mathrm{I}$ & 0,09 \\
\hline
\end{tabular}

Notas: ${ }^{* *}: \mathrm{p}<.0 \mathrm{I} ;{ }^{*}: \mathrm{p}<.05 ; \chi^{2}$ : Chi cuadrado; $\chi^{2} /$ Coef.: se indica Chi Cuadrado o Coeficiente de Contingencia según corresponda; rs: Coeficiente de Correlación de Spearman. Los números de contenidos corresponden con los de la Tabla 1. FuENTE: elaboración propia 
$\mathrm{Al}$ analizar la variable sexo se observa un mayor porcentaje de referencias a la articulación interinstitucional por parte de la mujeres (33\%) frente a los hombres $(19 \%)$, sin que exista una hipótesis para explicar esto. Por su parte, hay una significativa relación inversa entre la edad de los extensionistas y la mención a la gestión de grupos como parte del trabajo de ER, sin que se encuentre la misma relación en el caso de la experiencia. Esto sugiere que se trata de una diferencia generacional: los más jóvenes, con independencia de su experiencia, reconocen como más importante el tema que aquellos de mayor edad.

También se observa una relación positiva entre la experiencia en el trabajo de ER y la valoración de la participación y el reconocimiento de las necesidades y cultura de los productores, la concepción de la ER como transferencia de tecnologías y cambio de mentalidad de los productores, y el reconocimiento y valoración del saber campesino. Interesante esto, ya que cuestiones que parecerían contraponerse, como la valoración de la participación y el reconocimiento del saber campesino, por un lado, y una ER orientada a la transferencia de tecnologías (que supone rechazar el punto de vista y el saber hacer de la gente) por el otro, parecerían relacionarse todas ellas con mayor experiencia de los extensionistas.

$\mathrm{Al}$ analizar la relación entre la variable referida a la transferencia de tecnologías y la valoración de la participación y el reconocimiento del saber campesino, se observa una leve relación inversa, pero que no llega a ser estadísticamente significativa ( $r s=-.10, p=.14$ y rs=-.06, p=.36, respectivamente). No obstante, estos resultados sugieren que aun en el caso de personas que han estado más tiempo en la función de ER (lo que implica haber participado en aquellos periodos con mayor énfasis transferencista en Argentina), también han generado, a partir de su experiencia, un reconocimiento de la necesidad de tomar en cuenta el punto de vista y la situación de los beneficiarios, cuando se trabaja en ER, así como de sus saberes y capacidades productivas. Se sigue que los elementos que, a nivel conceptual, parecerían forman parte de modelos de ER diferentes, no necesariamente ocurren a nivel empírico, por lo que se presta atención a otras variables, como en este caso la experiencia laboral.

Los extensionistas que trabajan en diferentes instituciones también muestran un perfil diferencial en cuanto a su concepción de ER. Con respecto al trabajo interdisciplinario en equipos de ER, se observan más menciones en el caso de la SAF que en el resto de las instituciones ( $51 \%$ y $44 \%$ respectivamente, frente a $37 \%$ y $32 \%$ del INTA, y $26 \%$ y $19 \%$ del ProHuerta). La SAF es la institución con mayor porcentaje de extensionistas provenientes de profesiones no técnicas, muchos de ellos formados en ciencias sociales (23\% frente a $2 \%$ de INTA y $7 \%$ de ProHuerta). Esto genera la 
experiencia concreta del rol que puede desempeñar la interdisciplina y la formación de equipos de ER, lo que quizás indujo a mencionar y a valorar estas opciones.

Respecto de la concepción de la ER como capacitación de productores, se observa claramente una mayor presencia en el ProHuerta (29\%) frente al INTA (гі\%) y la SAF (го\%), si bien ProHuerta fue un programa orientado a la entrega de insumos biológicos acompañado de formación para su uso. Esto se complementa con la referencia a la ER como gestión de acciones participativas, poco presente en ProHuerta (6\%) pero más frecuente en INTA (21\%) y SAF (24\%).

En lo que implica al posicionamiento crítico respecto a qué es desarrollo, ER y saber científico, se observa un mayor porcentaje en el caso del INTA (32\%), seguido de la SAF (27\%) y luego ProHuerta (I2\%). En principio podría haberse esperado un mayor porcentaje en la SAF, al existir allí mayor presencia de profesionales del área social, más propensos a este tipo de reflexiones. Otra posible interpretación sería pensar que la variable institución haya enmascarado otra. Esto puede pensarse en relación con el nivel educativo, también vinculado con un mayor posicionamiento crítico en cuanto a las nociones de extensión y desarrollo. Es que la formación de posgrado es mucho más común en INTA (29.46\%; 8.58\% en ProHuerta y 13.15\% en la SAF). Así, podría pensarse que es el nivel educativo y no la institución, la que favorecería una actitud reflexiva y crítica al respecto.

En lo relativo a la región del país donde trabajan los encuestados, no se observa un perfil diferencial, pero sí en el caso del nivel educativo, asociado de modo positivo con la mayoría de los contenidos que dan forma a la concepción de ER de los encuestados. Visto esto, se podría formular como hipótesis que el nivel educativo es una variable particularmente relevante respecto de la concepción de ER de los encuestados. No obstante, al tomar consciencia de que las relaciones significativas, identificadas en este caso, son todas positivas, es decir, a mayor nivel educativo, más referencias a los diferentes contenidos, una explicación plausible podría ser: aquellos con mayor nivel educativo podrían tender a contestar con mayor amplitud a las preguntas, lo que favorecería más categorizaciones que redundarían en mayor presencia de los distintos contenidos. Ahora bien, según esta lógica, sería coherente pensar que los relevantes serían aquellos contenidos que no parecen estar asociados con esta variable. En cualquier caso, si bien no resulta clara la incidencia que la formación de los extensionistas tiene respecto de la concepción de ER, los datos sugieren que esta incidencia podría ser significativa.

Por otra parte, el título académico de los encuestados también se relaciona con la concepción de ER. En cuanto a la gestión y manejo de grupos en ER, se 
observan más menciones por parte de aquellos con formación en ciencias sociales (8I \%), y más bajo en los veterinarios (38\%), en personas no universitarias $(52 \%)$ y en agrónomos (60\%). Sobre la formación de equipos de ER, se observa mayor interés en los veterinarios $(56 \%)$ y en profesionales de las ciencias sociales ( $44 \%)$, frente a los ingenieros agrónomos $(29 \%)$ y los no universitarios (10\%).

En relación con la necesidad de herramientas para trabajar en ER, se destaca de nuevo la mirada de los veterinarios ( $44 \%)$, frente a los de ciencias sociales $(25 \%)$ y a los agrónomos (26\%), ubicados en un lugar intermedio, y muy pocas referencias en el caso de los que no poseen título universitario ( $16 \%$ ). Por último, entre quienes mencionan con más frecuencia el hecho de que los pequeños productores poseen una racionalidad diferente, se encuentran los de ciencias sociales $(50 \%)$, seguidos por los veterinarios $(25 \%)$ los agrónomos (26\%), y los no universitarios (10\%). Se concluye entonces que la formación específica de los extensionistas incide en su concepción de ER, aunque no necesariamente en el generar un perfil más o menos transferencista o dialógico.

\section{Conclusiones}

Como resultados más destacados de esta investigación pueden mencionarse los siguientes: en primer lugar, el perfil global de la concepción de ER de los encuestados no parece identificarse con ningún modelo teórico específico de ER. Es decir, los contenidos que nutren la concepción de ER de los encuestados no parecen identificarse con aquellos propuestos por ninguno de los cuatro modelos de extensión desarrollados en la Introducción. Segundo, respecto de las variables asociadas de modo estadístico con los contenidos de las concepciones de ER de los encuestados, cabe destacar, por importancia, la formación (nivel educativo y título universitario) y los años de experiencia de los extensionistas.

Tercero, también merecen un análisis más detallado tres contenidos de la concepción de ER de los encuestados: la importancia dada al trabajo grupal como parte del trabajo de ER, el bajo porcentaje de extensionistas que mencionan la gestión de procesos participativos como parte de su labor y la actitud crítica de casi un cuarto de los encuestados sobre su propia práctica.

En lo correspondiente a la falta de relación directa entre el perfil global de la concepción de ER de los encuestados y los contenidos de los modelos teóricos de ER, pueden generarse dos interpretaciones. Una sería que diferentes sujetos se identificarían con diferentes modelos, sin que exista primacía de ninguno. Sin duda, es 
posible encontrar en las encuestas casos de adscripciones claras a modelos específicos. No obstante, parece más plausible pensar que los extensionistas no tienden a concebir a la ER desde modelos teóricos coherentes, sino a partir de cierto sincretismo que puede articular elementos diversos (y aun contrapuestos) en una misma práctica, algo similar a lo observado en Paraguay (Landini, Bianqui y Crespi, 20I3). De esto deriva la necesidad de profundizar en la comprensión de esta diversidad y dejar se asumir que a nivel empírico existen los modelos descritos por la teoría.

En lo que concierne a las variables que inciden en la concepción de ER, se destaca la formación de los extensionistas. Si bien esto resulta evidente en los datos, son poco claros los mecanismos que explican esta relación. Podría arriesgarse que un mayor nivel educativo favorecería un modo de hacer ER que reconoce más la diversidad y los saberes de los productores, lo que favorece acciones menos transferencistas y más dialógicas o participativas, aunque esto debería ser evaluado en profundidad en el futuro.

Por su parte, los años de experiencia en extensión también parecen incidir en la concepción de ER. En este estudio se observó que a más años de experiencia, mayor reconocimiento del valor de la participación y de la importancia del saber campesino. Esto discute la idea de que, por tanto, los profesionales más jóvenes poseen un perfil más abierto y participativo, cuando en realidad su falta de experiencia podría favorecer posicionamientos más soberbios a partir de considerarse portadores de cierta "verdad", que debe ser implementada en terreno, ya sea a en el ámbito tecnológico o de modelos de desarrollo.

Respecto de los diferentes contenidos que dan forma a la concepción de ER de los encuestados, en primer lugar llama la atención la fuerte presencia del trabajo grupal como parte del quehacer de la ER, mas cuando la formación de los extensionistas suele tener un perfil técnico. Esto invita a repensar tanto la necesaria diversidad disciplinaria en el plantel institucional, como en la importancia de capacitar en esta área a quienes trabajan en terreno. En un plano más general, esto se relaciona con la existencia de una concepción de desarrollo amplia, no exclusivamente productivista, que aparece en un porcentaje significativo de encuestados.

Queda claro que quienes trabajan de manera directa con pequeños productores reconocen la complejidad de la realidad que enfrentan, y pareciera que esta no puede ser abordada únicamente con capacidades técnicas. De aquí se derivarían las referencias a la necesidad de abordajes interdisciplinarios, con preferencia en el contexto de la formación de equipos de ER. Este espacio podría permitir un trabajo reflexivo sobre la práctica, ámbito privilegiado para reflexionar y optar 
de manera consciente por los fines y prácticas de ER que se desea llevar adelante (Landini et al., 2013).

Al mismo tiempo también se destaca el contraste entre el bajo porcentaje de extensionistas que mencionan la gestión de procesos participativos como parte de su función y la importancia general dada a esta. Es como si la crítica a la falta de participación fuera algo que se asignara a los otros (sobre todo, instituciones), sin reconocer el papel (y el margen de maniobra) que los propios extensionistas podrían cumplir en este proceso.

Por último, también cabe analizar la actitud crítica identificada en casi un cuarto de los encuestados respecto de nociones básicas referidas al trabajo de ER, lo que muestra la existencia de profesionales con capacidad para pensar sobre su hacer. Esta potencialidad debería aprovecharse para poner en cuestión las prácticas de ER y los supuestos en que estas se basan, para generar opciones reflexivas y conscientes de los fines que se desean alcanzar y los mejores medios hacerlo. Se trata nada más de generar espacios para no seguir haciendo lo mismo cuando algo falla y buscar entender por qué se hace lo que se hace en un proceso de aprendizaje reflexivo. Así, sería posible trabajar de manera más activa desde las instituciones sobre el modelo de ER que se desea llevar adelante, y dejar de asumir que los principios discursivos planteados por estas son los que, en efecto, guían las prácticas.

\section{Referencias}

Alemany, C. y Sevilla, E. (2007). ¿Vuelve la extensión rural? Reflexiones y propuestas agroecológicas vinculadas con el retorno y fortalecimiento de la extensión rural en América Latina. Realidad Económica, (227), 52-74.

Cáceres, D. (1995). Estrategias campesinas en sociedades rurales contemporáneas. Revista de la Facultad de Agronomía, I5(I), 67-72.

Elcherothpops, G., Doise, W. y Reicher, S. (20II). On the Knowledge of Politics and the Politics of Knowledge: How a Social Representations Approach Helps Us Rethink the Subject of Political Psychology. Political Psychology, 32(5), 729-758.

Elgue, M. y Chiaradía, C. (2007). Formas asociativas para la agricultura familiar: elementos para el análisis funcional y normativo de las distintas formas jurídicas. Buenos Aires: Secretaría de Agricultura, Ganadería, Pesca y Alimentos.

Freire, P. (1973). ¿Extensión o comunicación? La concientización en el medio rural. Buenos Aires: Siglo XXI. 
Howarth, C. (2006). A Social Representation is not a Quiet Thing: Exploring the Critical Potential of Social Representations Theory. British Journal of Social Psychology, 45(I), 65-86.

Instituto Nacional de Tecnología Agropecuaria [INTA] (2004). El INTA que queremos. Plan Estratégico Institucional 2005-2015. Buenos Aires: Autor.

Landini, F. (2010). La dinámica de los saberes locales y el proceso de localización del saber científico. Algunos aportes desde un estudio de caso. Cuadernos de Desarrollo Rural, $7(65)$, 2I-45

Landini, F. (20II). Racionalidad económica campesina. Mundo Agrario, 23, versión electrónica.

Landini, F., Bianqui, V. y Crespi, M. (2013). Evaluación de las creencias sobre extensión rural de los extensionistas paraguayos. Psiencia, 5(I), 3-I4.

Landini, F., Bianqui, V. y Russo, M. (2013). Evaluación de un proceso de capacitación para extensionistas rurales implementado en Paraguay. Revista de Economia e Sociologia Rural, 5 I(supi), 9-30.

Landini, F., Leeuwis, C., Long, N. y Murtagh, S. (2014). Towards a Psychology of Rural Development. Journal of Community and Applied Social Psychology, 24(6), $534-546$

Landini, F., Long, N., Leeuwis, C. y Murtagh, S. (20I4). Theoretical Guidelines for a Psychology of Rural Development. Cuadernos de Desarrollo Rural, II(74), I25-I47.

Landini, F., Murtagh, S. y Lacanna, M. (2009). Aportes y reflexiones desde la psicología al trabajo de extensión con pequeños productores. Formosa: INTA

Leeuwis, C. (2004). Communication for Rural Innovation. Retbinking Agricultural Extension. Oxford: Blackwell.

Lobos, G. (2005). Micro-negocios asociativos campesinos: análisis económico de un sistema de producción ovina, región del Maule, Chile. Gestão y Produção, I2(2), I65-175.

Long, N. (2007). Sociología del desarrollo: una perspectiva centrada en el actor. México D. F.: Ciesas.

Machado, J., De Hegedüs, P. y Silveira, L. (2006). Estilos de relacionamento entre extensionistas e produtores. Ciência Rural, 36(2), 64I-647.

McLeod Rivera, W. y Qamar, M. (2003). Agricultural Extension, Rural Development and the Food Security Challenge. Roma: FAO.

Mora Delgado, J. (2008). Persistencia, conocimiento local y estrategias de vida en sociedades campesinas. Revista de Estudios Sociales, (29),I22-I33. 
Núñez, J. (2004). Los saberes campesinos: implicaciones para una educación rural. Investigación y Postgrado, 29(2), 13-60.

Patiño, J. (2000). Prácticas y racionalidad productiva. Estrategias de los Mazahuas de Ixtlahuaca. Convergencia, 7(22), 193-246.

Silvetti, F. y Cáceres, D. (1998). Una perspectiva sociohistórica de las estrategias campesinas del noreste de Córdoba, Argentina. Debate Agrario, (28), 103-127.

Stage, O. y Rekve, P. (1998). Food Security and Food Self-Sufficiency: the Economic Strategies of Peasants in Eastern Ethiopia. The European Journal of Development Research, I0(I), I89-200.

Tommasino, H., González, M., Guedes, E. y Prieto, M. (2006). Extensión crítica: los aportes de Paulo Freire. En H. Tommasino y P. De Hegedüs (Eds.), Extensión: reflexiones para la intervención en el medio urbano y rural (pp. I2I-135). Montevideo: Universidad de la República.

Tort, M. (2008). Enfoques de la extensión rural. En nuestro agro: ¿̇evolución, complementación u oposición? En J. Balsa, G. Mateo y M. Ospital (Comps.), Pasado y presente en el agro argentino (pp. 428-450). Buenos Aires: Lumiere.

Tort, M. y Román, M. (2005). Explotaciones familiares: diversidad de conceptos y criterios operativos. En M. González (Ed.), Productores familiares pampeanos: Hacia la comprensión de similitudes y diferenciaciones zonales (pp. 35-65). Buenos Aires: Astralib.

Tsakoumagkos, P., Soverna, S. y Craviotti, C. (2000). Campesinos y pequeños productores en las regiones agroeconómicas de Argentina. Buenos Aires: Ministerio de Economía. 